\title{
To what extent are psychiatrists aware of the comorbid somatic illnesses of their patients with serious mental illnesses? - a cross-sectional secondary data analysis
}

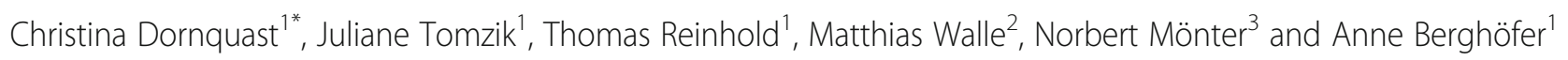

\begin{abstract}
Background: Somatic comorbidities are a serious problem in patients with severe mental illnesses. These comorbidities often remain undiagnosed for a long time. In Germany, physicians are not allowed to access patients' health insurance data and do not have routine access to documentation from other providers of health care. Against this background, the objective of this article was to investigate psychiatrists' knowledge of relevant somatic comorbidities in their patients with severe mental illnesses.

Methods: Cross-sectional secondary data analysis was performed using primary data from a prospective study evaluating a model of integrated care of patients with serious mental illnesses. The primary data were linked with claims data from health insurers. Patients' diagnoses were derived on the basis of the ICD-10 and the Anatomical Therapeutic Chemical (ATC) classification system. Diabetes, hypertension, coronary artery disease (CAD), hyperlipidaemia, glaucoma, osteoporosis, polyarthritis and chronic obstructive pulmonary disease (COPD) were selected for evaluation. We compared the number of diagnoses reported in the psychiatrists' clinical report forms with those in the health insurance data.

Results: The study evaluated records from 1,195 patients with severe mental illnesses. The frequency of documentation of hypertension ranged from $21 \%$ in claims data to $4 \%$ in psychiatrists' documentation, for COPD from 12 to $0 \%$, respectively, and for diabetes from 7 to $2 \%$, respectively. The percentage of diagnoses deduced from claims data but not documented by psychiatrists ranged from $68 \%$ for diabetes and $83 \%$ for hypertension, to 90\% for CAD to $98 \%$ for COPD.

Conclusions: The majority of psychiatrists participating in the integrated care programme were insufficiently aware of the somatic comorbidities of their patients. We support allowing physicians to access patients' entire medical records to increase their knowledge of patients' medical histories and, consequently, to increase the safety and quality of care.
\end{abstract}

Keywords: Mental disorders, Somatic comorbidity, Secondary data analysis, Claims data

\footnotetext{
* Correspondence: christina.dornquast@charite.de

${ }^{1}$ Institute for Social Medicine, Epidemiology and Health Economics, Charité -

Universitätsmedizin Berlin, Luisenstrasse 57, 10117 Berlin, Germany

Full list of author information is available at the end of the article
} 


\section{Background}

People with severe mental illnesses often have somatic comorbidities, meaning that one or more somatic diseases co-occur with the mental illness. In many cases, this phenomenon can be ascribed to a mutual dependence between the psychiatric illness and the somatic disease [1]. In fact, higher risks of getting a psychiatric disorder were shown for persons with somatic diseases [2]. On the other hand, also the risk of having a somatic disease is approximately twice as high for persons with severe mental illnesses than for people without mental disorders [3]. Thirty to fifty percent of patients experiencing psychiatric disorders have clinically relevant comorbid physical diseases [4], including cardiovascular disease, diabetes mellitus and respiratory and lung diseases [4-8]. In patients with severe mental illnesses, physical comorbidities often remain undiagnosed for a longer period of time than in those without severe mental illnesses [4, 9]. Many people with severe mental illnesses do not have or rarely consult a general practitioner and are confronted with various other barriers to receiving care [10-12].

Physicians working in the German health care system are not allowed to access patients' health insurance data and do not have routine access to documentation from other providers of health care, in contrast to, e.g., UK and Denmark. To obtain information on comorbid diseases and other health care usage data, physicians must interview the patient or his/her relatives and actively order medical notes from colleagues in other specialties. Physicians need to rely on the completeness of the information given by the patient. This increases the risk that psychiatrists are not aware of comorbidities in patients with severe mental illnesses, which is problematic, e.g., when prescribed psychotropic drugs interact with drugs prescribed for somatic diseases by other physicians [13-16].

In this paper, we focus on psychiatrists' awareness of their patients' somatic comorbidities. We use data from a German pre-post study that evaluated the costs of a model of integrated health care for patients with serious mental illnesses [17]. The integrated health care programme comprises complex outpatient treatment provided by, e.g., psychiatrists and psychiatric nurses, general practitioners, clinicians and social workers. Inclusion in the programme substituted for an otherwise necessary hospitalisation. The study reported insufficient documentation of common somatic diseases by the treating psychiatrists. The purpose of this article was to compare the presence of selected somatic diseases in patients with severe mental illnesses with the frequency of documentation of these diseases by the treating psychiatrists. The selected somatic diseases were diabetes, hypertension, coronary artery disease (CAD), hyperlipidaemia, glaucoma, osteoporosis, polyarthritis and chronic obstructive pulmonary disease (COPD).

\section{Methods}

Study design

We performed a cross-sectional secondary data analysis based on a prospective, multi-centre, non-controlled observational study of an integrated care model in mental health [17]. The study was approved by the institutional ethics committee of the Charité-Universitätsmedizin Berlin.

\section{Study population and data sources}

All patients who were insured by DAK-Gesundheit health insurance or a health insurance company belonging to the group BKK VAG Mid-Germany and who were newly included in the integrated health care programme between July 2007 and December 2009 in Berlin/Brandenburg and Lower Saxony were eligible to participate in this study. Detailed inclusion and exclusion criteria are reported elsewhere [17]. Briefly, patients were included in the integrated health care programme if they had an ICD-10 diagnosis within the range of F0.X to F8.X, showed seriously impaired social functioning as defined by a Global Assessment of Functioning (GAF) score of less than or equal to fifty, exhibited a severity score greater than or equal to five on the Clinical Global Impression (CGI) Scale and were at least 18 years old. Persons rated by the treating psychiatrist as having acute suicidal tendencies or a high likelihood of discontinuing treatment were excluded; the former were directly referred to inpatient treatment [17].

From a sample of 1,364 patients in the integrated care model, we created a subsample for the present analysis by excluding patients with BKK statutory health insurance $(n=145)$ due to lack of permission to use their data as well as patients without existing clinical report forms from their treating psychiatrist and health insurance company data $(n=24)$ (see Fig. 1$)$. The analysed subsample contained 1,195 participants. Psychiatrists' clinical report forms were available from July 2007 to December 2009. In contrast, health insurance data were available from 31 July 2003 (first entry) to 31 December 2009 (last entry).

Psychiatrists documented relevant study information on medical history and the clinical status of their patients in clinical report forms containing sociodemographic data, medical history, ICD-10 codes for current psychological and somatic diagnoses, GAF scores, CGI scores and specific psychopathological scales. The GAF is a numeric scale that evaluates the psychological, social and occupational functioning of adults [18]. The CGI scale describes the severity of an illness [19]. The main psychiatric diagnosis, which was documented by the psychiatrists, was defined as being the one that resulted in the patient's inclusion in the outpatient integrated care programme. Completeness of documentation was achieved as much as possible by a 


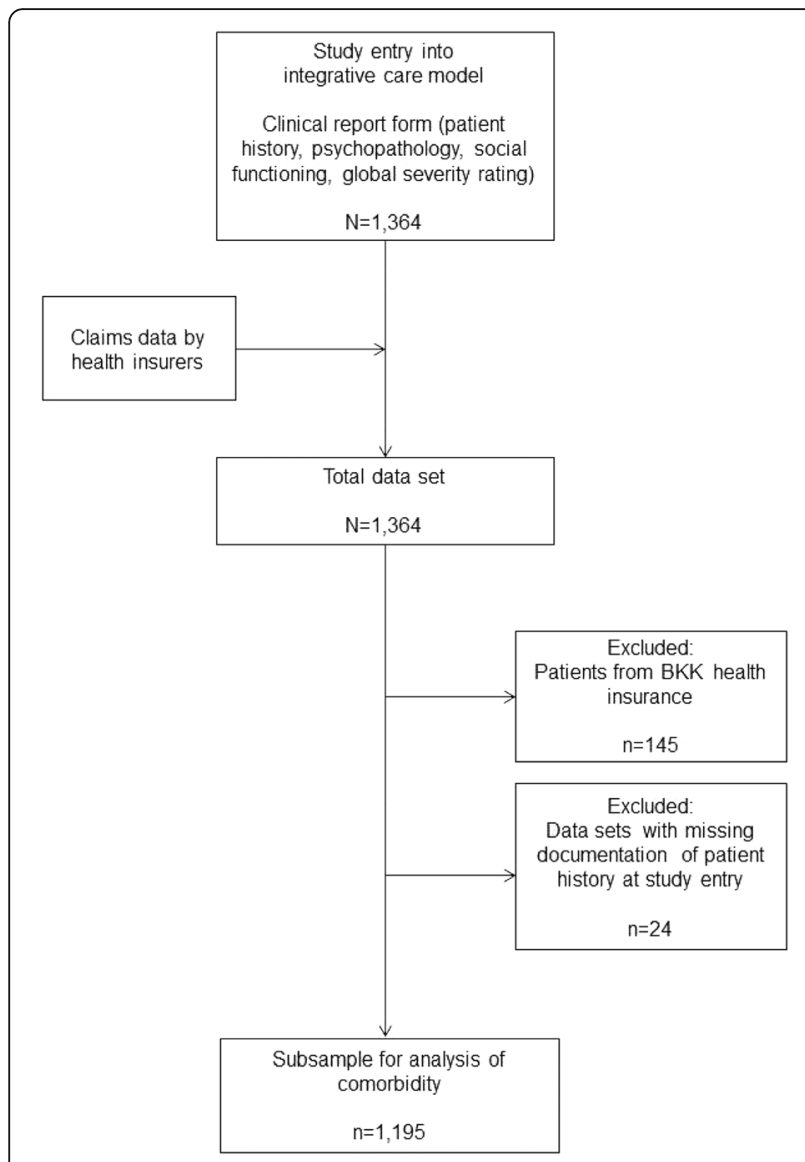

Fig. 1 Study design and data management

standardised query system used by the study personnel. If psychiatrists did not list any somatic comorbidities on a patient's clinical report form, we assumed that no somatic comorbidities were known to them.

\section{Deriving ICD-diagnoses from ATC-Codes}

The health insurance data contributed for each study participant comprised all Anatomical Therapeutic Chemical (ATC) classification codes for medications prescribed to the patient and ICD-10 codes if the patient was hospitalised, stayed at a rehabilitation centre or received an illness certificate. Somatic diagnoses were deduced either directly from the ICD-10 codes or indirectly from the medication regimen, as proposed by previous studies [20-24]. For our analysis, we selected highly prevalent somatic diseases with clinical relevance to patients with severe mental illnesses [1, 4, 25-27] or with an increased risk of severe interactions with common medications used for the treatment of severe mental illnesses [4, 13-16]. The selected somatic diseases were diabetes, hypertension, coronary artery disease (CAD), hyperlipidaemia, glaucoma, osteoporosis, polyarthritis and chronic obstructive pulmonary disease (COPD). Their respective ICD-10 codes and ATC codes for specifically prescribed drugs are listed in Table 1 . Somatic diseases that could not unequivocally be deduced from the ATC codes of the prescriptions, such as stroke, epilepsy or Parkinson's disease, were not considered, although they may be highly prevalent.

\section{Assessment of psychiatrists' knowledge}

For each selected disease, we counted the number of patients for whom psychiatrists documented the respective diagnosis, as well as those for whom a diagnosis was derived on the basis of the health insurance data. Percentages were calculated by dividing the respective numbers by $N=1,195$. To assess psychiatrists' knowledge of their patients' somatic diseases, the frequency of documentation of somatic diseases was descriptively compared using both psychiatrists' clinical report forms and health insurance data for the following three steps of analysis:

First, we determined the number of somatic diagnoses documented by psychiatrists that were also derived from the health insurance data. Second, we determined the number of diagnoses that were documented by psychiatrists but not documented in the health insurance data. Third, we determined the number of diagnoses reported solely in the health insurance data.

\section{Sensitivity analysis}

We performed a sensitivity analysis to verify the validity of our defined ATC code criteria for the detection of diseases. For each of the selected diseases, we investigated how many patients with an ICD-10 diagnosis were also identified by the defined ATC codes.

A descriptive statistical analysis was performed using $\mathrm{R}$ version 3.0.0 [28].

\section{Results}

\section{Baseline characteristics}

Table 2 shows the baseline characteristics of the study population. A total of 1,195 patients experiencing a psychiatric disorder, $69 \%$ of whom were females, were included in the study. The mean patient age at entry into the integrated care model was $48.0 \pm 16.1$ years. More than half of the patients had an affective disorder as their main psychiatric diagnosis. Schizophrenia, schizotypal and delusional disorders were documented as the main diagnoses in approximately one fifth of the patients.

\section{Psychiatrists' awareness of their patients' comorbidities}

The health insurance data contained 4,891 ICD-10 code entries and 96,951 ATC code entries for the 1,195 patients, of which $112(2 \%)$ and 4,973 (5\%), respectively, were missing, i.e., an insurance number and/or a date was listed without an ICD-10 or ATC code.

The number of patients for whom a diagnosis was documented by the psychiatrist and the number of 
Table 1 Predefined criteria for selected somatic diseases using ICD-10 and ATC codes for prescribed medications ${ }^{\mathrm{a}}$

\begin{tabular}{|c|c|c|}
\hline Disease & $\begin{array}{l}\text { ICD-10 code in health } \\
\text { insurance data }\end{array}$ & ATC code prescription documented in health insurance data \\
\hline Diabetes & E10-E14, G6320 & A10 (at least one time) \\
\hline Hypertension & $110-115$ & $\begin{array}{l}\text { C02 (at least twice within } 90 \text { days) OR } \\
\text { at least two different ATC codes [C02, C03, C07, C08 or C09] (within } 90 \text { days) }\end{array}$ \\
\hline CAD & $120-125$ & No distinct diagnosis by means of ATC codes \\
\hline Hyperlipidaemia & E780-E785 & C10 (at least one time) \\
\hline Glaucoma & $\mathrm{H} 40, \mathrm{H} 42$ & $\begin{array}{l}\text { S01E (at least twice within } 90 \text { days) AND manual review by a physician to identify } \\
\text { patterns and decide individually }\end{array}$ \\
\hline Osteoporosis & M80-M82 & At least two ATC codes [G03XC01, H05, M05BA or M05BB] (within 90 days) \\
\hline Polyarthritis & M05-M09 & $\begin{array}{l}\text { At least three ATC codes [M01B, M01CA, M01CB, M01CX, M02, L04AA13, L04AB01, } \\
\text { L04AB04 or L04AX03] (within } 180 \text { days) AND manual review by a physician to identify } \\
\text { patterns and decide individually }\end{array}$ \\
\hline COPD & $J 44, J 4200, J 4390$ & At least three ATC codes [R03, R05] (within 180 days) \\
\hline
\end{tabular}

aproposed by previous studies [20-24]

patients who were diagnosed on the basis of the health insurance data differed widely (see Table 3). The frequency of documentation in the psychiatrists' clinical report forms was very low, with hypertension (4\%) and diabetes (2\%) being the most commonly documented diseases. The frequency of documentation of the remaining diseases ranged from 0 to $1 \%$. When combining data from

Table 2 Baseline characteristics of the study population

\begin{tabular}{|c|c|c|}
\hline$N=1,195$ & Number & Percent \\
\hline Age (mean $\pm S D)$ & $48.0 \pm 16.1$ & \\
\hline \multicolumn{3}{|l|}{ Sex } \\
\hline Male & 366 & $31 \%$ \\
\hline Female & 829 & $69 \%$ \\
\hline \multicolumn{3}{|l|}{ Main psychiatric diagnosis ${ }^{a}$} \\
\hline $\begin{array}{l}\text { F0 Organic, including symptomatic, } \\
\text { mental disorders }\end{array}$ & 57 & $5 \%$ \\
\hline $\begin{array}{l}\text { F1 Mental and behavioural disorders } \\
\text { due to psychoactive substance use }\end{array}$ & 16 & $1 \%$ \\
\hline $\begin{array}{l}\text { F2 Schizophrenia, schizotypal and } \\
\text { delusional disorders }\end{array}$ & 234 & $20 \%$ \\
\hline F3 Mood [affective] disorders & 681 & $57 \%$ \\
\hline $\begin{array}{l}\text { F4 Neurotic, stress-related and } \\
\text { somatoform disorders }\end{array}$ & 137 & $12 \%$ \\
\hline F6 Disorders of adult personality and behaviour & 28 & $2 \%$ \\
\hline F8 Disorders of psychological development & 1 & $0 \%$ \\
\hline Information missing & 41 & $3 \%$ \\
\hline Clinical Global Impression $\left(\left.C G\right|^{b}\right)(\text { mean } \pm S D)^{a}$ & $5.3 \pm .7$ & \\
\hline $\begin{array}{l}\text { Global Assessment of Functioning }\left(\mathrm{GAF}^{\mathrm{C}}\right) \\
(\text { mean } \pm \mathrm{SD})^{\mathrm{a}}\end{array}$ & $36.7 \pm 8.8$ & \\
\hline
\end{tabular}

${ }^{a}$ These numbers are from the first quarter of inclusion in the integrated health care programme

${ }^{b}$ Possible scores for CGI range from 1 to 7 , with higher scores indicating more severe illness

'Possible scores for GAF range from 0 to 100, with higher scores indicating better psychological, social and occupational functioning health insurers and the psychiatrists' clinical report forms, hypertension was the most frequently diagnosed disease (23\%), followed by hyperlipidaemia (13\%), COPD (12\%) and diabetes (7\%). Distinctly lower frequencies were observed for glaucoma (3\%), CAD (3\%), osteoporosis $(2 \%)$, and polyarthritis (1\%). Of all the patients in this study, 728 (61\%) had no somatic comorbidities, and 272 (23\%), 123 $(10 \%)$ and $72(6 \%)$ had one, two or three or more somatic comorbidities, respectively.

When comparing diagnoses documented by psychiatrists and diagnoses derived from health insurance data, fewer than $30 \%$ of diagnoses were reported in both data sources (see Table 3). This percentage was less than $10 \%$ for hypertension, CAD, hyperlipidaemia, glaucoma, polyarthritis and COPD. Patients for whom a diagnosis was documented by a psychiatrist but not documented in health insurance data were infrequent (less than $10 \%$ of the diagnosed patients). The majority of diagnoses (68 to 98\%) were identified in the health insurance data but not documented by psychiatrists.

\section{Sensitivity analysis}

We investigated how many patients with an ICD-10 code in their health insurance data were also diagnosed by ATC codes. For the majority of diseases, 52 to $75 \%$ were diagnosed by both codes (see Table 4). However, none of the four cases of polyarthritis diagnosed by ICD-10 code could be identified by the ATC criteria.

\section{Discussion}

Our analysis showed that psychiatrists' documentation of somatic comorbidities in their patients was very low. The percentage of diagnoses that were documented in health insurance data but not by psychiatrists ranged from $68 \%$ for diabetes to $98 \%$ for COPD. This constitutes a problem, as many patients within our selected study population also 


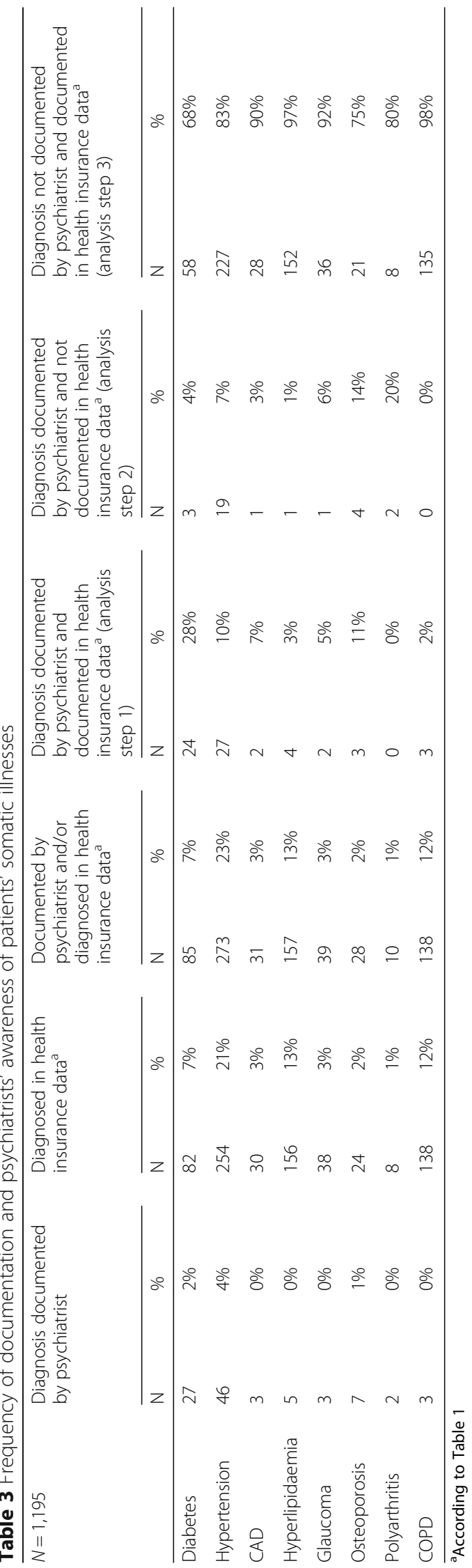


Table 4 Sensitivity analysis

\begin{tabular}{llll}
\hline$N=1,195$ & Diagnosed by ICD-10 code (in health insurance data) & \multicolumn{2}{c}{$\begin{array}{l}\text { Patients with somatic diagnoses derived from ATC criteria who also } \\
\text { had documented ICD-10 codes in the health insurance data (in \%) }\end{array}$} \\
\cline { 3 - 5 } & $N$ & $N$ & 8 \\
\hline Diabetes & 12 & 25 & $67 \%$ \\
Hypertension & 48 & n.a. ${ }^{\text {a }}$ & $52 \%$ \\
CAD & 0 & 0 & $0 \%$ \\
Hyperlipidaemia & 0 & 3 & $60 \%$ \\
Glaucoma & 5 & 3 & $75 \%$ \\
Osteoporosis & 4 & 0 & $0 \%$ \\
Polyarthritis & 4 & 10 & $53 \%$ \\
COPD & 19 & & 0
\end{tabular}

${ }^{a}$ CAD patients were solely diagnosed by ICD- 10 codes

suffer from somatic diseases. The number of patients with documentation of the selected somatic diseases ranged from $1 \%$ for polyarthritis to $23 \%$ for hypertension.

One of the main limitations of this study was the fact that relevant diagnoses could be missed in the health insurance data, as shown by our sensitivity analysis (see Table 4). However, we only had access to ICD-10 codes reported to a health insurer from hospitalisations, stays at rehabilitation centres and illness certificates. Data from the Association of Statutory Health Insurance accredited Physicians (Kassenärztliche Vereinigung), which also contain ICD-10 codes originating from the outpatient sector, as well as data from general practitioners, were not available. Instead, we derived somatic diagnoses from the ATC codes of prescribed drugs. This method is more prone to omission and misclassification of disease. Various diseases are difficult or impossible to identify on the basis of ATC codes, e.g., CAD, heart failure and pain syndromes.

We may have underestimated the frequency of somatic diseases in this sample because a proportion of the patients studied may not have been treated for their somatic diseases and therefore would not have ATC codes in their health insurance data, and some patients were missing data from their health insurer. We expect that either technical problems or changes in health insurers are responsible for missing data, and hence we assume that selection bias is not a problem. Another limitation of our study is that, for certain patients, specific ATC codes were documented at the end of the period for which data were available. These patients were likely not assigned a diagnosis because our definition of diagnoses required several documentations of ATC codes within a certain time period. Additionally, the different time frames of the health insurance data and psychiatrists' documentation obtained must be mentioned. However, these different time frames do not represent a serious constraint, as the selected somatic diseases are all chronic or lifelong diseases.
Our work is also limited by the fact that we could not discern why so many diagnoses were not documented by psychiatrists. It is possible that a psychiatrist was aware of a disease but did not document it in the clinical report form. However, this is unlikely due to the elicitation of this information in the context of a study, and a careful query process used to request non-completed answers.

Our data stem from a specialised treatment setting. However, the quality of documentation for the included patients is expected to be even better, due to the research setting in which it was gathered, than it would be in a typical treatment setting. The quality of the routine data from the health insurance company that were added to the primary data did not differ between patients inside or outside integrated care models, as the documented data are largely stipulated by the Germany Social Insurance Code.

In conclusion, we do not see this as a limitation to the validity of our results.

Previous studies have shown that many somatic diseases in patients with severe mental illnesses remain undetected or undiagnosed by their psychiatrists. A study conducted in 1989 reported that psychiatrists documented comorbidities in their patients in only $53 \%$ of cases [9]. In our analysis, diabetes was the only disease to be consistently documented by psychiatrists. This may be because it is particularly important for psychiatrists to know that their patients have been diagnosed with diabetes, as atypical antipsychotics have a diabetogenic effect. Additionally, patients usually have a diabetic ID card that can be shown to the psychiatrist. Such ID cards comprise amongst others information about the patients' prescription schedule, dates of routine medicals checks and current blood levels of laboratory indicators. Furthermore, diabetes is a highly prevalent disease, and psychiatrists may be more aware of this disease and may ask about it more frequently.

Patients with severe mental illnesses are reported to be less likely to self-report their medical conditions to a 
physician. They are also less likely to report that their treating physician informed them about a co-occurring somatic disease [29].

Finally, the frequency of documented somatic diseases in our study population differed from those documented by other studies. The CATIE-study from 2005 reported a prevalence of hypertension of $27 \%$ for patients with schizophrenia [30], and hypertension was the most frequently diagnosed cardiovascular comorbidity in these patients. We found similar results in our sample with hypertension as most frequent documented disease (23\%). Additionally, the authors of the CATIE-study reported a prevalence of diabetes of $13 \%$ in patients with schizophrenia, which is almost twice as high as the frequency of documentation we calculated [30]. The frequency of somatic diseases we calculated is expected to be lower because patients with other serious mental illnesses were included in addition to patients experiencing schizophrenia. Those patients are often treated with atypical antipsychotics, which are known to have diabetogenic effects. Other studies reported similar prevalences as the CATIE-study [25, 31]. A study from 2004 reported a prevalence of COPD of $23 \%$ in patients with severe mental illnesses [32]. This proportion is almost twice as high as the frequency we calculated in our sample (12\%). This discrepancy is most likely explained by the fact that we derived diagnoses from the ATC codes of prescribed drugs. We were therefore more likely to miss relevant diagnoses.

\section{Conclusions}

Many patients experiencing mental illnesses also have somatic diseases. These comorbidities pose challenges to the management of the mental, medical and personal health of a patient. Our data show that psychiatrists participating in the integrated care programme were insufficiently aware of the somatic comorbidities of their patients with psychiatric disorders. It can be assumed that outside of a research setting the level of awareness may be even lower.

Improved communication among physicians with distinct specialties is desirable to provide optimal therapy for a patient. However, the implementation of improved communication should not be the responsibility of the physician alone. Physicians have heavy workloads and may not be able to initiate an exchange of information with colleagues who are treating the same patient. Improved communication should be initiated and promoted by administrative institutions through the use of financial incentives or facilitated access to patients' treatment data (e.g., through the use of an electronic health card). We support the idea of allowing physicians to access patients' entire medical records to increase their knowledge of patients' medical history and, consequently, to increase the safety and quality of care.
Psychiatrists should also be aware that the treatment of somatic diseases can affect psychiatric diseases and a patient's response to psychiatric treatment. Hence, psychiatrists should obtain a thorough somatic medical history in their patient interviews. Because patients experiencing psychiatric disorders often have significant somatic comorbidities, and because of the associated health and economic consequences, additional studies, such as interview-based assessments of psychiatrists' awareness of somatic comorbidities with the verification of true and false positives by independent assessors, are needed to investigate this issue.

\section{Abbreviations}

ATC: Anatomical Therapeutic Chemical; CAD: Coronary artery disease; CGI: Clinical Global Impression; COPD: Chronic obstructive pulmonary disease; GAF: Global Assessment of Functioning

\section{Acknowledgements}

We thank American Journal Experts (AJE) for English language editing.

Funding

No funding was obtained for this investigator-initiated study.

\section{Availability of data and materials}

Data will not be shared due to restriction by the German Statutory Health Insurance System.

\section{Authors' contributions \\ $C D$ participated in the design of the study and drafted the initial manuscript. JT participated in the design of the study, performed the data analysis and helped to draft the manuscript. TR participated in the design of the study, reviewed and revised the manuscript. MW acquired data, reviewed and revised the manuscript. NM acquired data, reviewed and revised the manuscript. $A B$ made substantial contributions to the acquisition of data, participated in the design of the study, reviewed and revised the manuscript. All authors read and approved the final manuscript.}

\section{Competing interests}

CD, JT and TR declare that they have no competing interests. MW and NM received payment from the statutory health insurance company DAK Gesundheit in the context of integrated care. $A B$ previously received funding from the statutory health insurance company DAK Gesundheit for evaluating integrative care using statutory health insurance data. Claims data were provided by DAK Gesundheit, Hamburg, Germany.

\section{Consent for publication}

Not applicable.

\section{Ethics approval and consent to participate}

The prospective, multi-centre, non-controlled observational study of an integrated care model was approved by the institutional ethics committee of the Charité-Universitätsmedizin Berlin (No. EA1/088/08). There has been written consent to participate from all participants. The cross-sectional secondary data analysis of claims data did not need formal ethics approval complying with national guidelines (Amtsblatt 34/2004, §2 Abs.1)

\section{Author details}

${ }^{1}$ Institute for Social Medicine, Epidemiology and Health Economics, Charité Universitätsmedizin Berlin, Luisenstrasse 57, 10117 Berlin, Germany. 2IVPNetworks GmbH, Lübecker Str. 126, 22087 Hamburg, Germany. ${ }^{3}$ PIBB GmbH \& Co. KG - Psychiatrie Initiative Berlin Brandenburg, Tegeler Weg 4, 10589 Berlin, Germany. 
Received: 7 November 2015 Accepted: 21 February 2017 Published online: 23 February 2017

\section{References}

1. Baumeister $\mathrm{H}$, Härter M. Körperliche Beschwerden bei Patienten mit psychischen Störungen. Z Psychiatr Psychol Psychother. 2007;55(1):9-19.

2. Wells KB, Golding JM, Burnam MA. Affective, substance use, and anxiety disorders in persons with arthritis, diabetes, heart disease, high blood pressure, or chronic lung conditions. Gen Hosp Psychiatry. 1989;11(5):320-7.

3. Wells KB, Golding JM, Burnam MA. Chronic medical conditions in a sample of the general population with anxiety, affective, and substance use disorders. Am J Psychiatr. 1989;146(11):1440-6.

4. Hewer W, FüeßI H. Körperliche Erkrankungen bei psychiatrischen PatientenTeil I. Fortschritte der Neurologie. Psychiatrie. 2009;77(12):720-37.

5. Horvitz-Lennon M, Kilbourne AM, Pincus HA. From silos to bridges: meeting the general health care needs of adults with severe mental illnesses. Health Aff. 2006;25(3):659-69.

6. Kilbourne AM, Morden NE, Austin K, Ilgen M, McCarthy JF, Dalack G, Blow FC. Excess heart-disease-related mortality in a national study of patients with mental disorders: identifying modifiable risk factors. Gen Hosp Psychiatry. 2009:31(6):555-63.

7. Osborn DP, Nazareth I, King MB. Risk for coronary heart disease in people with severe mental illness Cross-sectional comparative study in primary care. Br J Psychiatry. 2006;188(3):271-7.

8. Osborn DP, Levy G, Nazareth I, Petersen I, Islam A, King MB. Relative risk of cardiovascular and cancer mortality in people with severe mental illness from the United Kingdom's General Practice Research Database. Arch Gen Psychiatry. 2007;64(2):242-9.

9. Koran LM, Sox H, Marton K, Moltzen S, Sox C, Kraemer H, Imai K, Kelsey T, Rose T, Levin L. Medical evaluation of psychiatric patients. Arch Gen Psychiatry. 1989;46(8):733-40.

10. van Hasselt FM, Schorr SG, Mookhoek EJ, Brouwers JR, Loonen AJ, Taxis K. Gaps in health care for the somatic health of outpatients with severe mental illness. Int J Ment Health Nurs. 2013;22(3):249-55.

11. Druss BG. Improving medical care for persons with serious mental illness: challenges and solutions. J Clin Psychiatry. 2007;68 Suppl 4:40-4.

12. Vreeland B. Bridging the gap between mental and physical health: $A$ multidisciplinary approach. J Clin Psychiatry. 2007;68 Suppl 4:26-33.

13. Sandson NB, Armstrong SC, Cozza KL. An overview of psychotropic drug-drug interactions. Psychosomatics. 2005;46(5):464-94.

14. Spina E, Santoro V, D'Arrigo C. Clinically relevant pharmacokinetic drug interactions with second-generation antidepressants: an update. Clin Ther. 2008;30(7):1206-27.

15. Guo JJ, Wu J, Kelton CM, Jing Y, Fan H, Keck PE, Patel NC. Exposure to potentially dangerous drug-drug interactions involving antipsychotics. Psychiatr Serv. 2012;63:1080-8.

16. Ostermann JK, Berghöfer A, Andersohn F, Fischer F. Frequency and clinical relevance of potential cytochrome P450 drug interactions in a psychiatric patient population. BMC Health Serv Res. 2016;16:482.

17. Fischer F, Hoffmann K, Mönter N, Walle M, Beneke R, Negenborn S, Weinmann S, Willich S, Berghöfer A. Kostenevaluation eines Modells der Integrierten Versorgung für schwer psychisch Kranke. Das Gesundheitswesen. 2014;76(02):86-95.

18. Wittchen $\mathrm{H}$, Saß H, Zaudig M, Koehler K. Diagnostische Kriterien und Differentialdiagnosen des diagnostischen und statistischen Manuals psychischer Störungen DSM III-R Basel. Weinheim: Beltz; 1989.

19. Weyer G. Internationale Skalen für Psychiatrie. Göttingen: Beltz Test; 2005.

20. Chini F, Pezzotti P, Orzella L, Borgia P, Guasticchi G. Can we use the pharmacy data to estimate the prevalence of chronic conditions? a comparison of multiple data sources. BMC Public Health. 2011;11(1):688.

21. Halfon P, Eggli $Y$, Decollogny A, Seker E. Disease identification based on ambulatory drugs dispensation and in-hospital ICD-10 diagnoses: a comparison. BMC Health Serv Res. 2013;13(1):453.

22. Kuo RN, Dong Y-H, Liu J-P, Chang C-H, Shau W-Y, Lai M-S. Predicting healthcare utilization using a pharmacy-based metric with the WHO's anatomic therapeutic chemical algorithm. Med Care. 2011;49(11):1031-9.

23. Lamers LM, van Vliet RC. The pharmacy-based cost group model: validating and adjusting the classification of medications for chronic conditions to the Dutch situation. Health Policy. 2004;68(1):113-21.
24. Fransoo R, Martens P, The Need to Know Team, H P, C B, I K, A B, E A. The 2013 RHA Indicators Atlas. In: Winnipeg MV, editor. Manitoba Centre for Health Policy. 2013.

25. Hert M, Correll CU, Bobes J, Cetkovich-Bakmas M, Cohen D, Asai I, Detraux J, Gautam S, Möller HJ, Ndetei DM. Physical illness in patients with severe mental disorders. I. Prevalence, impact of medications and disparities in health care. World Psychiatry. 2011;10(1):52-77.

26. Lederbogen F, Kopf D, Hewer W. Interdisziplinäre Station für psychisch Kranke mit erheblichen somatischen Komorbiditäten. Nervenarzt. 2008;79(9): 1051-8.

27. Lambert TJ, Velakoulis D, Pantelis C. Medical comorbidity in schizophrenia. Med J Aust. 2003;178(9):S67.

28. R Core Team. R: A Language and Environment for Statistical Computing. 2013

29. Kilbourne AM, McCarthy JF, Welsh D, Blow F. Recognition of co-occurring medical conditions among patients with serious mental illness. J Nerv Ment Dis. 2006;194(8):598-602.

30. Goff DC, Sullivan LM, McEvoy JP, Meyer JM, Nasrallah HA, Daumit GL, Lamberti S, D'Agostino RB, Stroup TS, Davis S. A comparison of ten-year cardiac risk estimates in schizophrenia patients from the CATIE study and matched controls. Schizophr Res. 2005;80(1):45-53.

31. Parks J, Svendsen D, Singer P, Foti ME, Mauer B. Morbidity and mortality in people with serious mental illness. VA, USA: National Association of State Mental Health Program Directors (NASMHPD) Medical Directors Council Alexandria; 2006.

32. Himelhoch S, Lehman A, Kreyenbuhl J, Daumit G, Brown C, Dixon L. Prevalence of chronic obstructive pulmonary disease among those with serious mental illness. Am J Psychiatr. 2004;161(12):2317-9.

\section{Submit your next manuscript to BioMed Central and we will help you at every step:}

- We accept pre-submission inquiries

- Our selector tool helps you to find the most relevant journal

- We provide round the clock customer support

- Convenient online submission

- Thorough peer review

- Inclusion in PubMed and all major indexing services

- Maximum visibility for your research

Submit your manuscript at www.biomedcentral.com/submit

) Biomed Central 